\title{
Excitation of Vibrational Eigenstates of Coupled Microcantilevers using Ultrasound Radiation Force
}

\author{
Thomas M. Huber \\ Department of Physics \\ Gustavus Adolphus College \\ Saint Peter, MN 56082 \\ huber@gac.edu
}

\author{
Eric T. Ofstad \\ Department of Physics \\ Gustavus Adolphus College \\ Saint Peter, MN 56082 \\ eofstad@gac.edu
}

\author{
Samuel M. Barthell \\ Department of Physics \\ Gustavus Adolphus College \\ Saint Peter, MN 56082 \\ sbarthel@gac.edu
}

\author{
Arvind Raman \\ Department of Mechanical Engineering \\ Birck Nanotechnology Center \\ Purdue University \\ West Lafayette, IN 47907 \\ raman@ purdue.edu
}

\author{
Matthew Spletzer \\ Department of Mechanical Engineering \\ Purdue University \\ West Lafayette, IN 47907 \\ mspletzer@purdue.edu
}

\begin{abstract}
A coupled pair of $500 \mu \mathrm{m}$ length microcantilevers was excited using the ultrasound radiation force. The excitation was produced using the difference frequency between the two sidebands of a doublesideband suppressed carrier AM (DSB-SC-AM) waveform centered on $500 \mathrm{kHz}$ that was emitted by a focused ultrasound transducer. A laser Doppler vibrometer measured the frequency response and deflection shapes of the cantilever pair. The measured frequencies of the symmetric and antisymmetric eigenstates of the first transverse mode at $10 \mathrm{kHz}$ excited using the ultrasound radiation force were consistent with frequencies measured using a scanning-probe microscopy system. The ultrasound radiation force was also used to excite the symmetric and antisymmetric eigenstates of the $60 \mathrm{kHz}$ second transverse and $86 \mathrm{kHz}$ first torsional modes. These results demonstrate the capability of using the ultrasound radiation force for excitation of structures in air that are significantly smaller, and with higher resonance frequencies, than in any previous study.
\end{abstract}

\section{NOMENCLATURE}

$c \quad$ Speed of sound in air

$d_{r}(\boldsymbol{r}) \quad$ Drag coefficient on object at location $\boldsymbol{r}$

$e_{\Delta \mathrm{f}}(r, \mathrm{t}) \quad$ Instantaneous energy density at frequency $\Delta f$ at location $\boldsymbol{r}$ at time $t$

$f_{1}, f_{2} \quad$ Two ultrasound frequency components emitted by ultrasound transducer

$\Delta f \quad$ Difference frequency between ultrasound components: the frequency of the radiation force

$F_{\Delta \mathrm{f}}(\boldsymbol{r}, \mathrm{t}) \quad$ Instantaneous radiation force at frequency $\Delta f$ at location $\boldsymbol{r}$ at time $t$

$P(\boldsymbol{r}) \quad$ Amplitude of the ultrasound pressure field incident at position $\boldsymbol{r}$

$p(\boldsymbol{r}, \mathrm{t}) \quad$ Instantaneous pressure at location $\boldsymbol{r}$ and time $t$

$\Delta(\boldsymbol{r})$ Phase difference between frequency components $f_{1}$ and $f_{2}$ at position $\boldsymbol{r}$ Density of air

\section{INTRODUCTION}

Detection systems based on microcantilever sensors have recently emerged as a potential technology for the swift and precise recognition of chemical[1,2] or biological agents[3]. In most cases, this is accomplished by coating the cantilevers with substances which bind to the target molecules, effecting a change in cantilever mass and therefore the resonance frequency. For these applications, the cantilevers are often driven with base excitation, where, for example, a piezoelectric or similar material causes vibrations of the base of the structure. There are a limited number of non-contact methods that can be used for excitation, one of which is a time-varying electrostatic force can be used. While the optical radiation force is not sufficient for the microcantilever pair used in the current experiment, microcantilevers an order of magnitude smaller have been excited using a modulated laser[4]. 
The results described below demonstrate that it is possible to use ultrasound radiation force to excite the vibrational modes of a microcantilever array. With this technique, there is no contact between the cantilever and ultrasound source, so there is no mass loading. Another advantage of ultrasound excitation is that it is relatively insensitive to resonance frequencies of the fixture used to hold the sample.[5]

The device used in this experiment is a coupled pair of microcantilevers.[6] In previous studies, it was shown that Anderson (vibration) localization[7] could be used as a sensitive technique to detect masses on the order of $150 \mathrm{pg}$ added to one of the cantilevers; this was accomplished by measuring the amplitude change of the coupled cantilevers when mass was added instead of measuring their shift in resonance frequency. The cantilever array used had two eigenstates for each vibrational mode; a symmetric state where the two cantilevers oscillated in phase, and an asymmetric state, with a slightly higher frequency, where the two cantilevers vibrated out of phase. In the experiment described below, ultrasound radiation force was used to excite the lowest three pairs of vibrational modes of this coupled system.

\section{THEORY}

Previous papers have described in detail the mechanism for ultrasound stimulated excitation, both in air [5] and in water.[8] If an object is ensonified with a pair of ultrasound frequencies, $f_{1}$ and $f_{2}$, interference between the two frequencies produces a radiation force that results in a vibration of the object at the difference frequency $\Delta f=f_{2}-f_{1}$. In the measurements described below, both frequency components were emitted from a single transducer using a double-sideband suppressed-carrier amplitude modulated (DSB-SC-AM) waveform.[9] The two different ultrasound frequencies $f_{1}=f_{\mathrm{c}}-\Delta f / 2$ and $f_{2}=f_{\mathrm{c}}+\Delta f / 2$ were symmetrical about a central frequency $f_{\mathrm{c}}$. In the course of the experiment, the difference frequency $\Delta f$ was swept through a range of frequencies, and the response was measured at each frequency. If the radiation force at frequency $\Delta f$ corresponds to one of the resonance frequencies of the cantilever array, it will induce a larger amplitude vibration that will be detected using the laser Doppler vibrometer as shown in Figure 1.

The radiation force[10] is caused by changes in the energy density of an acoustic field. The ultrasound emitted by the transducer is a superposition of two waves of frequencies $f_{1}$ and $f_{2}$ with equal amplitudes $P(\boldsymbol{r})$. As the waves traverse the distance between the transducer and the arrival point $\boldsymbol{r}$, they arrive with phases ${ }_{1}(\boldsymbol{r})$ and ${ }_{2}(\boldsymbol{r})$, thus the pressure field $p(\boldsymbol{r}, \mathrm{t})$ due to the superimposed frequency components may be written as

$p(\boldsymbol{r}, \mathrm{t})=P(\boldsymbol{r}) \cos \left[2 f_{1} t+{ }_{1}(\boldsymbol{r})\right]+P(\boldsymbol{r}) \cos \left[2 f_{2} t+{ }_{2}(\boldsymbol{r})\right] .(1)$

This causes an instantaneous energy density given by $e(\boldsymbol{r}, t)=p(\boldsymbol{r}, t)^{2} / c^{2}$; this energy density will have a time- independent component, a component at the difference frequency $\Delta f$, and high-frequency components at multiples of $f_{1}$ and $f_{2}$. The radiation force of interest for the current technique is the energy density component at the difference frequency, which can be written as

$$
e_{\Delta f}(\boldsymbol{r}, t)=\frac{P(\boldsymbol{r})^{2}}{c^{2}} \cos [(2 \Delta f) t+\Delta(\boldsymbol{r})]
$$

Assuming that $P(\boldsymbol{r})$ is a plane wave, this will impart a force in the beam direction on an object of area $d S$ with drag coefficient $d_{r}(\boldsymbol{r})$ given by [5]

$$
\begin{aligned}
F_{\Delta f}(\boldsymbol{r}, t) & =e_{\Delta f}(\boldsymbol{r}, t) d_{r}(\boldsymbol{r}) d S \\
& =\frac{P(\boldsymbol{r})^{2}}{c^{2}} \cos [(2 \Delta f) t+\Delta(\boldsymbol{r})] d_{r}(\boldsymbol{r}) d S
\end{aligned}
$$

The total radiation force as a function of time is the integral of Equation 3 over the entire surface of the object; this radiation force can induce a vibration of the object at a frequency $\Delta f$. Vibration due to this radiation force is a function of the size, shape and mechanical impedance of the object. Previous studies have shown that this radiation force can be used for modal analysis of a variety of systems[11] including pipe organ reeds,[5] hard-drive suspensions,[12] MEMS mirrors and gyroscopes.[13]

\section{EXPERIMENTAL SETUP}

The coupled microcantilever array (Fig. 2) used in this experiment consisted of two nearly identical gold cantilevers each nominally measuring $500 \mu \mathrm{m}$ in length, $100 \mu \mathrm{m}$ in width and $10 \mu \mathrm{m}$ thick, protruding from an overhang with a width of $90 \mu \mathrm{m}$. The cantilevers were separated by $250 \mu \mathrm{m}$.[6] Because of the overhang, there is a coupling between the cantilevers, and thus they can be modeled as a pair of coupled oscillators. Because of this coupling, each of the vibrational modes of a simple cantilever will be split into symmetric and antisymmetric eigenstates. If the masses of the two cantilevers are identical, the amplitude of these eigenstates would be the same. However, if there is a difference in mass, it breaks the symmetry between the two eigenstates, leading to a difference in amplitude.[14]

The measurements of cantilever vibration were taken using a Polytec OFV-3000 laser Doppler vibrometer with the OFV-502 head mounted on a 2-dimensional translation stage system. By using the series of mirrors and lenses shown in Figure 1, this vibrometer was adapted so that the measurement point could be focused to a spot roughly $10 \mu \mathrm{m}$ across that could be directed to any portion of the cantilever array. For the purpose of optimizing measurement amplitudes, the measurements were usually taken near the free ends of the cantilevers. To determine operational deflection shapes, measurements of amplitude and phase of the deflection at many points on the surface were imported into Vibrant Technology ME'Scope VES software. 
A single Ultran NCG500-D25-P76 transducer was used. This transducer has a central frequency of $500 \mathrm{kHz}$, and a bandwidth of over $200 \mathrm{kHz}$; this transducer was used to excite resonances of the cantilever array up to $90 \mathrm{kHz}$. The focus diameter of this transducer was roughly $3 \mathrm{~mm}$, so the entire $0.5 \mathrm{~mm}$ cantilever array was roughly uniformly ensonified. The DSB-SC-AM signal used for excitation was produced by first generating a swept sinusoidal waveform, with frequencies on the order of 4.5-45 kHz using a National Instruments NI-USB-6251 Data Acquisition unit; this unit had an analog output sampling rate of 1 MSamples/sec. This waveform was directed to the AM modulation input of a Hewlett Packard 33120A function generator which has a $20 \mathrm{MHz}$ bandwidth. The output was a DSB-SC-AM waveform with a central frequency of $500 \mathrm{kHz}$ and sidebands separated by $\Delta f$ of $9 \mathrm{kHz}$ to $90 \mathrm{kHz}$ that was essentially free from all sampling artifacts. The waveforms were amplified using an ENI-2100 RF amplifier. Effects due to reflection from room walls or other surfaces that would lead to ultrasound interference were minimal because of the short focal length of the transducer used..

\section{RESULTS}

Figure 3 shows the frequency response measured when the ultrasound modulation of the $500 \mathrm{kHz}$ signal was swept from $\Delta f=9.9 \mathrm{kHz}$ to $10.3 \mathrm{kHz}$ during a 0.4 second chirp (with an averaging of 25 chirps). The square symbols show the velocity spectrum when the vibrometer measured the motion of a single point towards the end of one of the cantilevers; these are listed in arbitrary units because the vibrometer system has not been calibrated using the auxiliary lens and microscope objective system shown in Figure 1. This plot shows two resonance frequencies of the cantilever pair. By measuring the deflection shapes, it was verified that the lower frequency at $10.00 \mathrm{kHz}$ corresponded to the symmetric state, and the upper frequency at $10.17 \mathrm{kHz}$ corresponded to the antisymmetric state of the two cantilevers. Animation of these measured deflection shapes can be found online.[15] With the ultrasound signal turned off, the ambient excitation of the $10 \mathrm{kHz}$ peak was less than an order of magnitude smaller than the observed peak.

The results obtained for this pair of resonances of the cantilever array using ultrasound excitation are very similar to measurements made using a Nanotec Electronica scanningprobe microscopy system utilizing conventional base excitation. The data set from the scanning-probe microscope system was scaled to the same peak amplitude as the response from the ultrasound excitation, and is shown as diamonds on Figure 3. Both the resonance frequency and $Q$ value of the symmetric state were nearly identical for both techniques. For the antisymmetric state, the two techniques had a shift of about $20 \mathrm{~Hz}$ in the peak of the frequency response, and the relative amplitudes were different using the two techniques. These differences are not surprising, considering that completely different excitation methods were used, and since this state is very sensitive to minor variations in the excitation, measurement or masses of the cantilevers. Changes of this order of magnitude could be obtained by moving the measurement point used for the vibrometer during ultrasound excitation.

A finite element model (using Comsol Multiphysics) of the microcantilever system was developed by starting with the nominal dimensions of the cantilever and overhang. The dimensions of the overhang was modified from its nominal 90 $\mu \mathrm{m}$ width to $128 \mu \mathrm{m}$, and the thickness was increased from a nominal $10 \mu \mathrm{m}$ to $11 \mu \mathrm{m}$ until the simulated frequency response reproduced the frequency response of Figure 3. The model was then used to predict the frequencies at which the second transverse and first torsional modes would occur, along with the splitting of the symmetric and antisymmetric states for both of these modes. The ultrasound excitation frequencies were swept near these predicted frequencies; the experimentally measured frequencies were within a few percent of the frequencies predicted by the finite element model. The simulation also demonstrated differences in the amplitude of the symmetric and antisymmetric states of each mode that are consistent with the measurements shown in Figure 3-5.

Figure 4 shows the measured symmetric and antisymmetric second transverse states. To produce this graph, the DSB-SCAM signal was chirped from $\Delta f=58-62 \mathrm{kHz}$. The frequencies were identified as the symmetric $(59.2 \mathrm{kHz})$ and antisymmetric $(60.2 \mathrm{kHz})$ states by measuring the deflection shapes;[15] these deflection shapes and frequencies agree with the predictions of the simulation of about $59 \mathrm{kHz}$ and $60 \mathrm{kHz}$.

Figure 5 shows the first torsional modes of the array. Instead of using a chirped waveform, the transducer was excited with a DSB-SC-AM waveform with a constant difference frequency, and the vibrometer signal was sent to a Stanford Research Systems SR830 lock-in amplifier. The excitation frequencies were stepped from $\Delta f=84-91 \mathrm{kHz}$, and the amplitude and phase were measured at each frequency. To measure the symmetry characteristics of the two frequencies, the phase was measured at different locations on the two cantilevers at their resonance frequencies of $86.0 \mathrm{kHz}$ and $88.4 \mathrm{kHz}$. The $86.0 \mathrm{kHz}$ resonance exhibits a symmetry about the midpoint of the two cantilevers, whereas the $88.4 \mathrm{kHz}$ resonance has the left and right sides of the cantilevers twisting in phase. These frequencies are consistent with the predictions of the simulation of about 89 $\mathrm{kHz}$ and $92 \mathrm{kHz}$ respectively.

\section{CONCLUSIONS}

Taken as a group, Figures 3-5 demonstrate that it is feasible to use the ultrasound radiation force to excite resonances in a microcantilever system with dimensions on the order of 100's of microns. These microcantilevers are significantly smaller than any structure that has previously been excited using the ultrasound radiation force in air, a $750 \mu \mathrm{m}$ square MEMS gyroscope.[13] Since the feasibility of exciting these 
microcantilevers has been demonstrated, future studies will focus on whether it is possible to perform selective excitation of different symmetric or antisymmetric using a pair of ultrasound transducers,[13] a technique that cannot be accomplished using conventional base excitation. Future tests will also study whether this ultrasound excitation technique will allow greater sensitivity of mass differences than can be accomplished using conventional base excitation.

\section{ACKNOWLEDGEMENTS}

The authors appreciate discussions with James Greenleaf and Mostafa Fatemi of the Ultrasound Research Lab at the Mayo Clinic and for their loan of the Ultran ultrasound transducer used in this experiment. This material is based upon work supported by the National Science Foundation under Grant No. 0509993. Any opinions, findings and conclusions or recommendations expressed in this material are those of the author(s) and do not necessarily reflect the views of the National Science Foundation (NSF).

\section{REFERENCES}

[1] Rogers, L. Manning, M. Jones, T. Sulchek, K. Murray, B. Beneschott, J. D. Adams, Z. Hu, T. Thundat, H. Cavazos, and S. C. Minne, 2003, "Mercury vapor detection with a selfsensing, resonating piezoelectric cantilever," Rev. Sci. Instrum. 74, pp. 4899-4901.

[2] Pinnaduwage, L.A., Wig, A., Hedden, D.L., Gehl, A., Yi, D., Thundat, T., and Lareau, R.T., 2004, "Detection of trinitrotoluene via deflagration on a microcantilever," J. Appl. Phys. 95, pp. 5871-5875.

[3] Ilic, B., Yang, Y., and Craighead, H. G., 2004, "Virus detection using nanoelectromechanical devices.” Appl. Phys. Lett. 85, pp. 2604-2606.

[4] Ilic, B., Krylov, S., Aubin, K., Reichenbach, R., and Craighead, H. G., "Optical excitation of nanoelectromechanical oscillators," Appl. Phys. Lett. 86, 193114.

[5] Huber, T.M., Fatemi, M., Kinnick, R.R. and Greenleaf, J.F., 2006, "Noncontact modal analysis of a pipe organ reed using airborne ultrasound stimulated vibrometry," J. Acoust. Soc. Am. 119, pp. 2476-2482.

[6] Spletzer, M., Raman, A., Wu, A.Q., Xu, X., and Reifenberger, R., 2006, "Ultrasensitive mass sensing using mode localization in coupled microcantilevers," Appl. Phys. Lett. 88, 254102.

[7] Anderson, P.W., 1958, "Absence of diffusion in certain random lattices," Phys. Rev. 109, pp. 1492-1505.

[8] Fatemi, M. and Greenleaf, J.F., 1998, "Ultrasound stimulated vibro-acoustic spectography," Science 28, pp. 82-85.
[9] Chen, S., Fatemi, M., Kinnick, R.R. and Greenleaf, J.F., 2004, "Comparison of stress field forming methods for vibroacoustography," IEEE Trans. Ultrason., Ferroelect., Freq. Contr. 51(3), pp. 313-321.

[10] Westervelt, P.J., 1951, "Theory of steady force caused by sound waves," J. Acoust. Soc. Am. 23, pp. 312-315.

[11] Fatemi, M. and Greenleaf, J.F., 2001, "Mode excitation and imaging by the radiation force of ultrasound," J. Acoust. Soc. Am. 111, p. 2472.

[12] Huber, T.M., Calhoun, D., Fatemi, M., Kinnick, R.R., and Greenleaf, J.F., Noncontact modal testing of hard-drive suspensions using ultrasound radiation force, Proceedings of International Modal Analysis Conference (IMAC XXIV) February 2, 2006, Paper 363; see http://physics.gac.edu/ Huber/Presentations/imac_2006_februa ry/363_hub.pdf.

[13] Huber, T.M., Hagemeyer S.D., Ofstad, E.T., Fatemi, M., Kinnick, R.R., and Greenleaf, J.F., "Noncontact mode excitation of small structures in air using ultrasound radiation force," in Proceedings of 2007 Society for Experimental Mechanics Annual Conference, June 4, 2007, Springfield, MA Paper 400; see http://physics.gac.edu/ huber/presentations/sem_2007_june/40 0 hub.pdf

[14] Courant, R. and Hilbert, D., Methods of Mathematical Physics (Interscience, New York, 1953), Vol. 1.

[15] For animations of the modes of this system, see http://physics.gac.edu/ huber/animations/microcantilevers/

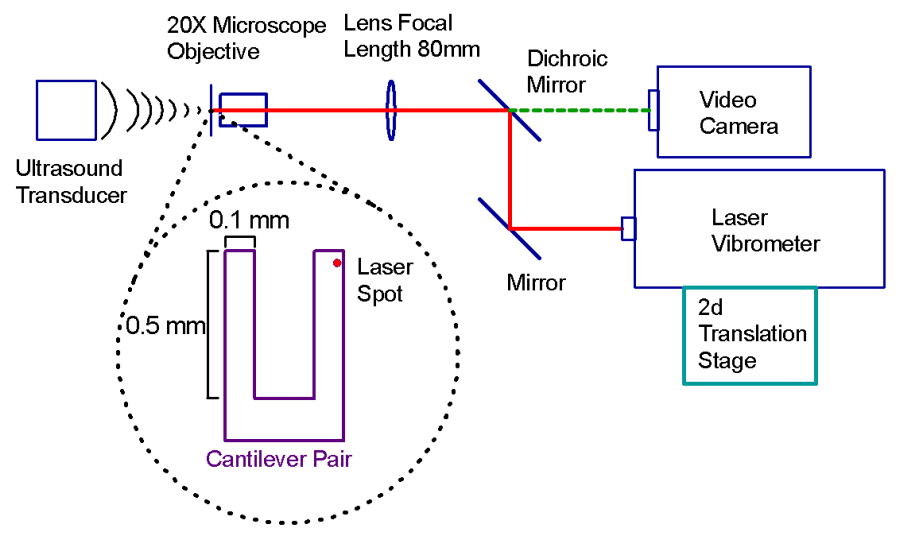

Figure 1: Diagram of apparatus used for ultrasound radiation force excitation of a microcantilever array. The velocity was measured using a laser Doppler vibrometer that was directed through a microscope objective to focus on the array. The dichroic mirror and video camera allowed imaging of the cantilever and laser spot. 


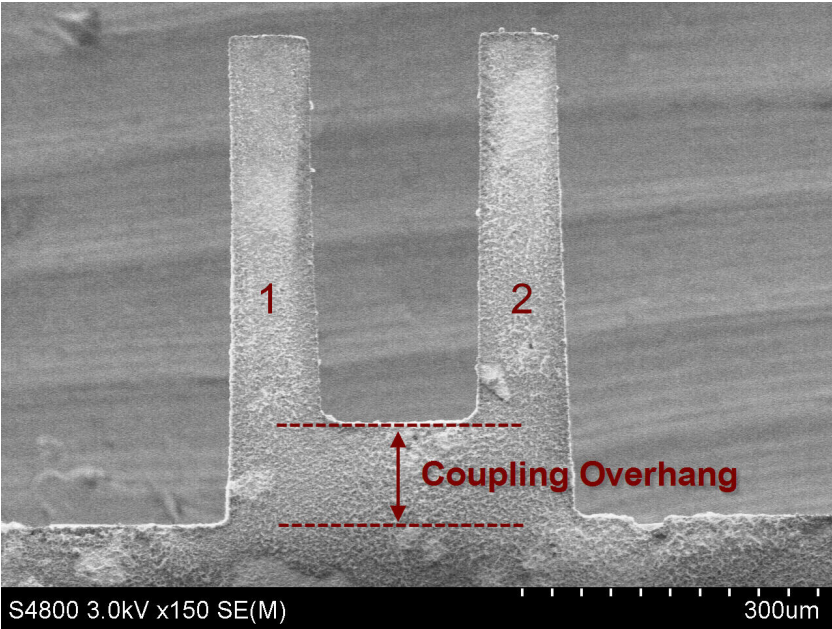

Figure 2: Scanning electron microscope (SEM) image of the $500 \mu \mathrm{m}$ length gold cantilevers used for this experiment. The overhang provides a coupling between the cantilevers.

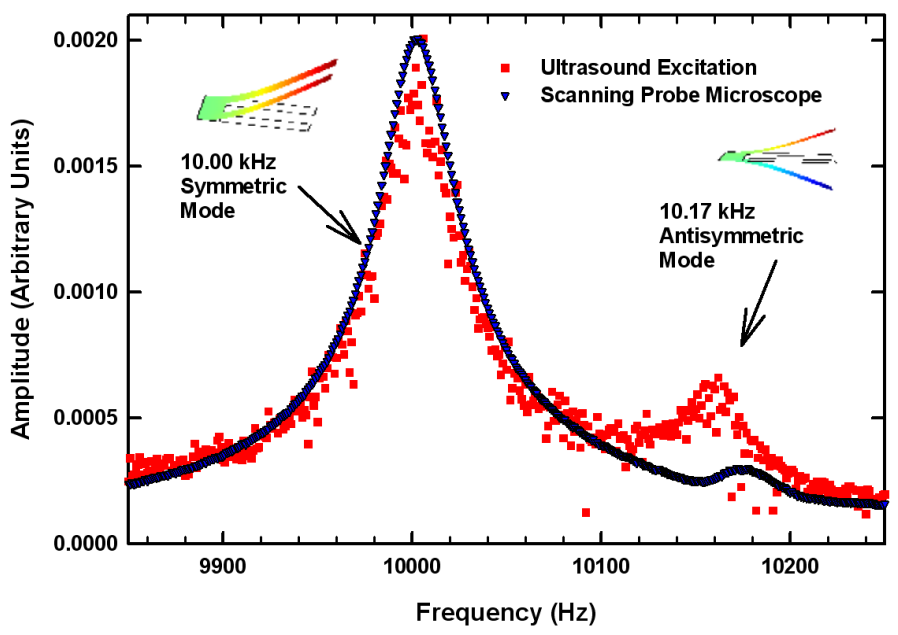

Figure 3: Velocity spectrum measured using laser Doppler vibrometer for microcantilever array excited with ultrasound radiation force (solid red squares), compared with conventional base excitation and measurement using a scanning probe microscope system (blue diamonds). The diagrams next to the resonances indicate the symmetric and antisymmetric states of the pair of cantilevers.

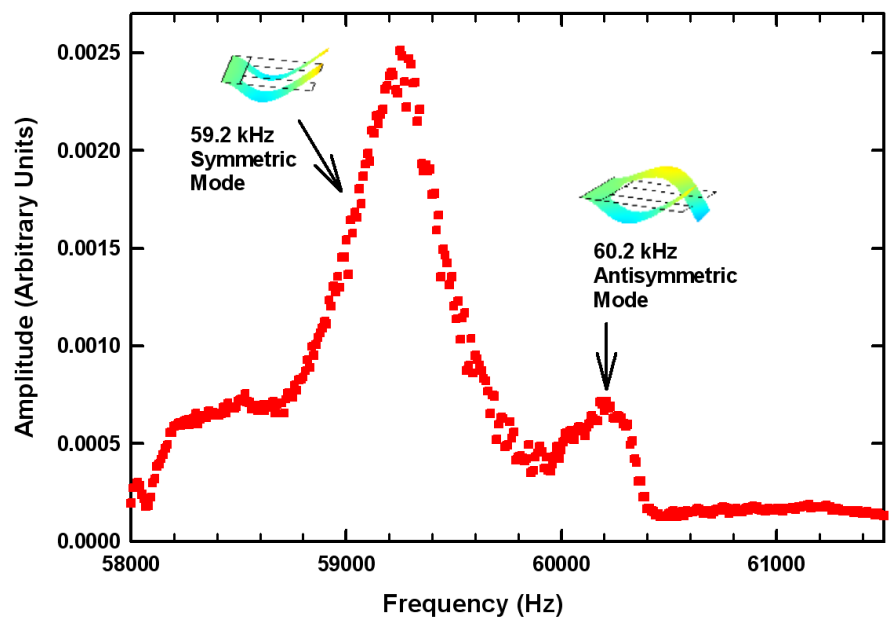

Figure 4: Velocity spectrum of symmetric and antisymmetric states for the $2^{\text {nd }}$ transverse mode of the microcantilever array excited using ultrasound radiation force.

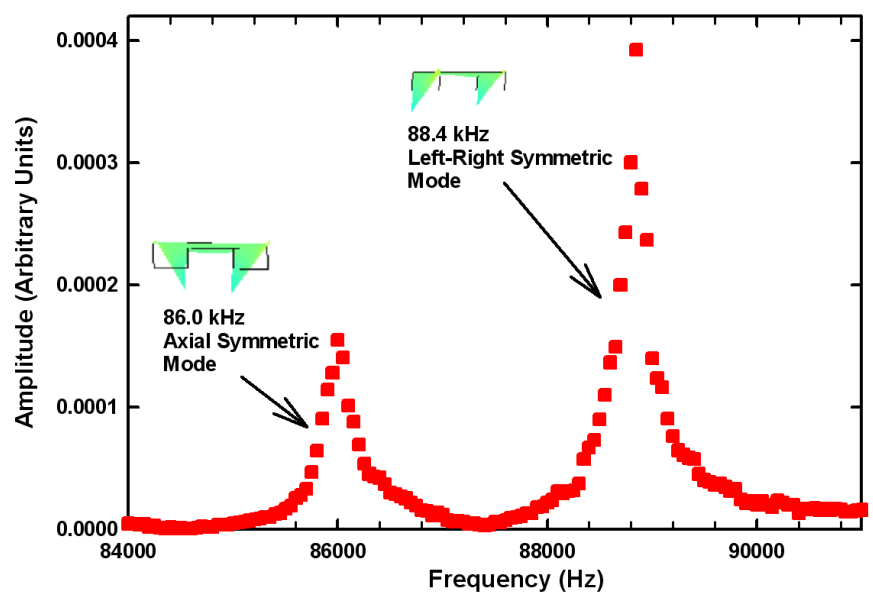

Figure 5: Velocity spectrum of the torsional modes of the microcantilever array. The lower eigenstate at $86.0 \mathrm{kHz}$ has the inner and outer edges of the two cantilevers vibrating in phase. The upper eigenstate has the left and right edges of the cantilevers moving in phase. 